\title{
IPAssess: A Protocol-Based Fingerprinting Model for Device Identification in the loT
}

This paper was downloaded from TechRxiv (https://www.techrxiv.org).

\section{LICENSE}

CC BY 4.0

SUBMISSION DATE / POSTED DATE

$14-10-2021 / 18-10-2021$

\section{CITATION}

Bhattacharyya, Siddhartha; Ganeriwala, Parth; Nandanwar, Shreya; Muthalagu, Raja; gupta, anubhav (2021): IPAssess: A Protocol-Based Fingerprinting Model for Device Identification in the loT. TechRxiv. Preprint. https://doi.org/10.36227/techrxiv.16815232.v1

$\mathrm{DOI}$

10.36227/techrxiv.16815232.v1 


\title{
IPAssess: A Protocol-Based Fingerprinting Model for Device Identification in the IoT
}

\author{
Parth Ganeriwala, Shreya Nandanwar, Anubhav Gupta, Dr. Siddharth Bhattacharyya, Dr. Raja Muthalagu
}

\begin{abstract}
Internet of Things (IoT) are the most commonly used devices today, that provide services that have become widely prevalent. With their success and growing need, the number of threats and attacks against IoT devices and services have been increasing exponentially. With the increase in knowledge of IoT related threats and adequate monitoring technologies, the potential to detect these threats is becoming a reality. There have been various studies consisting of fingerprinting based approaches on device identification but none have taken into account the full protocol spectrum. IPAssess is a novel fingerprinting based model which takes a feature set based on the correlation between the device characteristics and the protocols and then applies various machine learning models to perform device identification and classification. We have also used aggregation and augmentation to enhance the algorithm. In our experimental study, IPAssess performs IoT device identification with a $99.6 \%$ classification accuracy.
\end{abstract}

Index Terms-Cybersecurity, Explainable AI, Machine Learning, IoT Fingerprinting, Packet Data, Network traffic.

\section{INTRODUCTION}

$\mathbf{T}$ HERE are 7 billion IoT devices currently in use according to the data provided by the IoT Analytics conducted by Jing et al. [1]. This figure itself indicates the exponential growth of the devices and hence the growing need for securing the devices way before any attack is possible.

In order to deal with the security issues of IoT devices, one has to identify the type of the particular device present in the network. The device type plays an important role in the assessment as it helps to identify the existence of a device that might have restrictive policies associated it, restricting the amount or type of data which should be shared with it. If the device is exceeding its data boundaries, then it should be put in a quarantine network, a security breach should be identified and network rectification should be implemented. The device type identification also helps in recognizing the devices which might be malicious or pose a threat to other IoT devices or should not be present in that particular network.

Several approaches have been proposed in the literature for identifying the devices in the network. Shahid et al. [2] used machine learning models like Random Forest(RF) Classifier along with a t-SEN technique, to differentiate the network traffic generated by various IoT devices. This machine learning based approach achieved an overall accuracy as high as $99.9 \%$ on their test set. These kinds of models classify the devices on the basis of the size of the packets sent and received and the packet's inter-arrival times. These features play a key role in the identification and recognition of an IoT device in a network. Agarwal et al. [3] have used a web interface model and developed novel tools: "NetScanIoT" and "WebIoT Detection (WID)" which has achieved $92.45 \%$ accuracy in device type identification. An alternative approach adopted by Mavrogiorgou et al. [4] was implemented wherein a preexisting textual dataset was used and then the proposed model performed feature extraction on the images of the present IoT devices through object detection, thereby achieving classification.

Previous studies have investigated the various problems in the field of IoT device security based on their interactions over the network. Forensic analysis of the network has always been a salient approach in diagnosing the problem. As a result, there have been various studies which have performed in depth analysis on the network traffic generated by IoT devices. After recording traffic telemetry, the packet data has then been analysed to classify the state of the IoT devices. Furthermore, the behavior of IoT devices on the network has been monitored using a combination of Software Defined Networking (SDN) telemetry and machine learning methods which was conducted by Sivanathan et al. [5]. Meidan et al. [6] applied machine learning algorithms on traffic data for accurate identification of the IoT devices connected to a network. Alrashdi et al. [7] tried to detect anomalies of IoT cyberattacks in smart cities using machine learning yielding a $99.34 \%$ classification accuracy rate . In a recent study, a system for automated classification of device characteristics based on their network traffic, called System IDentifier (SysID) was introduced by Aksoy et al. [8]. It used various popular machine learning algorithms to have a completely automated way of classifying IoT devices using their TCP/IP packets without expert input for classification. In an experimental study with 23 IoT devices, SysID identified the device type from a single packet with over $95 \%$ accuracy - Common datasets were used to analyse the accuracy of the fingerprinting techniques and it was observed that the accuracy of device genre classification was $99.37 \%$ and the accuracy in the identification of an individual device from IoT Sentinel [9] dataset was found to be $83.35 \%$. However, the University of New South Wales (UNSW) [10] dataset yielded a device type identification accuracy of up to $97.78 \%$. Bai et al. [11] proposed a framework to group devices based on their semantic type (e.g., camera, fitness/medical device, environmental sensor). Grouping of various devices under a particular label potentially results in a model with a diverse range of performance boundaries since often various devices of a given type (e.g., cameras from different manufacturers) distinctly differ in their network behaviour. Therefore, models with diversity in categories of devices would yield high rate of false classification during testing phase. 
In our study, we try to work on these limitations by introducing our proposed modified approach. We form different feature sets based on previous studies and propose our own feature set based on the correlation of the features used in the IoT devices. We survey five different machine learning algorithms on the basis of classification accuracy by testing on the aforementioned feature sets. Augmentation and aggregation is performed on the original dataset to attain a more denser valued dataset. Device type identification and classification is then performed on the augmented dataset with packet aggregation. The contributions of this paper can be pointed out as follows:

- Comparison of different feature sets formed from the IoT Sentinel [9] dataset. We have found that some feature sets give a high accuracy even with comparatively less amount of features present, which helps us explain the correlation between the various IoT device protocols.

- Comparison of different machine learning algorithms based on of its classification accuracy and the time taken to identify the IoT devices.

- Classification of the different IoT devices based on packet aggregation, by enhancing the dataset using augmentation.

- Comparison of the performance of classifying models by aggregating devices with common labels to further reduce the time and complexity.

- Development of a prediction based model on the basis of the optimal machine learning model and the proposed feature set.

This paper would be organised in the following manner. Section II reviews the related works, Section III outlines the proposed methodology which has been split up into two parts: one describing how we chose a particular feature set along with the machine algorithm, and the second explaining our modified approach using augmentation and aggregation. Section IV reports the experimental results and are then discussed. Finally, Section V concludes our work.

\section{RELATED WORKS}

This section reviews the previous studies conducted in the field of IoT device identification. There have been many studies done on device fingerprinting techniques for IoT device classification, but their applicability is very controversial as they are based mostly on the physical or application layer without having taken in account the entire protocol spectrum. Hence, we focus here on research that is based on network packet behaviour which adheres to the entire protocol spectrum and includes the necessary information from all the different layers. One of the initial studies to use network packet features to perform IoT device identification and classification based on a fingerprinting method is IoT Sentinel [9]. This study uses the network flow to identify vulnerable devices in the network and isolate them from the user network by quarantining them. Their dataset consisted of a collection of data from 31 devices which they collected during the device installation phase which was repeated 20 times for each device. Each device was then fingerprinted based on 23 features (see Fig. 2) which were extracted from each of the first 12 packets for every device, resulting in a fingerprint comprised of 276 values. However, these 12 packets do not exactly represent flow but they are sequential packets from the same MAC address. Further, each device which was connected to the network is identified by these fingerprints and if any vulnerability is detected, the particular device is taken to the quarantine network and its connection to other devices, the local network, and the internet is restricted. This method is useful for identifying devices because the 23 features it creates are compressed/representative features: four of which are integers and the others binaryvalued. According to this study, 17 of 27 device types were detected with an identification accuracy of above $95 \%$, and 10 with an accuracy of around 50\% using RF (RF).

IoTSense [9] is another study which uses selected features of IoT Sentinel based on their own model assessment. IoTSense chooses 17 protocol-based features of the IoT Sentinel study which reflect device behaviour and they also add three payload-related features (see Fig. 2). This feature set is applied to five packets for each device to produce a 100-member fingerprint. According, to this study, the per device recall resulted to be $93-100 \%$ and an average accuracy of $99 \%$ were achieved using Extra Gradient Boosting (XGB), Decision Tree (DT) and k-Nearest Neighbours $(\mathrm{kNN})$. While some comparisons are made with the work of IoT Sentinel [9], the evaluation of IoTSense used a much smaller number of devices (i.e., 10 vs. 31). In addition, the IoTSense experiment set began with 14 devices, though only 10 devices were used for the evaluation as four devices did not produce sufficient data for the analysis approach used.

One of the most recent studies, is a novel fingerprinting method, IoTDevID [12], for device identification that uses machine learning to model the behaviour of IoT devices based on network packets. Their method uses an enhanced combination of features based on IoT Sentinel [9] and IoT Sense [13] and includes an approach for dealing with unbalanced device data via data augmentation. They have further demonstrated how to enhance device identification via a groupwise data aggregation and provide a comparative evaluation of the method against two recent identification methods using three public IoT datasets which together contain data from over 100 devices. They use a feature set based on the IoT Sentinel [9] feature set consisting of 23 features and additionally merge in 2 features from the IoT Sense [13] feature set to conjointly form their own: four of which are integers and the others binary-valued. In their fingerprinting process, they use individual packets instead of the entire network flow due to there being no standard size for the same. They use an aggregation algorithm to group packets together and then evaluate them by the machine learning algorithms. According to this study, they have F1-scores above $99 \%$ for all the datasets they have tested on, with considerable improvement gained from data aggregation.

\section{Proposed Methodology}

In this section, we elaborate on our proposed methodology shown in Figure 1, which is a step-by-step approach used 
to classify the IoT devices with improved efficiency, as a result reducing the time to achieve the desired accuracy of prediction. To begin with, it is essential to identify relevant feature sets that will give comparatively higher accuracy. In order to achieve this, we identify the important protocols which are highly correlated with the network traffic dataset and thus pre-process the data and formulate multiple feature sets, which is shown as Data pre-processing stage in the framework (see Fig 1). The next stage of our novel approach (Feature Set Selection Fig 1) is an experimental analysis to observe the optimal machine learning algorithm which has the highest accuracy and best time against the selected feature sets. The selected feature set and the optimal machine learning algorithm are then used in our proposed method of augmentation and aggregation which will be further discussed upon in the last two subsections respectively.

\section{A. Dataset}

After reviewing various papers, we came across several datasets but most of them were local network experiments with negligible amount of data for our methodology to be implemented on. We decided to choose the IoT Sentinel [9] Dataset due to vast amount of network traffic data and the appropriate features present, which are necessary in order to identify and classify a particular device along with the type of network packets it transmits over the network and its associated attributes.

The dataset consists of network traffic data collected from 31 smart home IoT devices which are of 27 different types, out of which 4 types are represented only by 2 devices each. The setup for each device was repeated atleast 20 times. This setup is represented in each directory in the form of a uniform collection of packet capture (pcap) files. The file_iotdevicemac.txt contains the MAC address of the corresponding IoT device.

The 26 features originally present in IoT Sentinel Dataset are destination IP counter (IP_add_count) (int), source port number (Portcl_src) (int), destination port number (Portcl_dst) (int), packet size (Pck_size) (int), packet raw data (Pck_rawdata), padding (IP_padding), router alert (IP_ralert), ARP, LLC, IP, ICMP, ICMPv6, EAPoL, TCP, UDP, Size of TCP width (TCP_w_size), HTTP, HTTPS, DHCP, BOOTP, SSDP, DNS, MDNS, NTP, Payload length (payload_l) and Entropy.

We start classifying the device type identification on the basis of Application protocol, Network layer protocol, Data Link layer, Transport layer and Physical Layer using a parsing script in Python which converts the pcap files and parses it to csv files based on the different protocols that have been defined above. This opens a scope for further research in the field of how one can use other types of protocol features to recognise and classify the IoT device more efficiently. Apart from just the protocols, we also focus on the packet size and the number of packets coming from each device, which give additional classification accuracy.
TABLE I

Classification of the port numbers used

\begin{tabular}{cc}
\hline Port Numbers & Equivalents \\
\hline \hline No Port & 0 \\
\hline Well Known Ports (between 0 and 1023) & 1 \\
\hline Registered Ports (between 1024 and 49151) & 2 \\
\hline Dynamic Ports (between 49152 and 65535) & 3 \\
\hline
\end{tabular}

\section{B. Data Pre-processing}

The Data pre-processing workflow, begins with the process of extraction of the feature sets from the pcap files. Using inbuilt libraries in python such as scapy and pandas, we discover the pcap extension files in the pcaps folder which contains the network data from the IoT_Sentinel dataset, and we display and categorize the ports which have been used during the network capture process of the devices mentioned, as displayed in Table I.

We then list the pcap files to be processed and match them with their particular MAC addresses. We calculate the payload entropy values for each of the devices given in the dataset. In 1948, Shannon [14] developed a measure for the uncertainty of a message. This measure is known as entropy in information theory. Shannon considers the case where we have a fixed number $\mathrm{m}$ of possible events $\mathrm{A}_{1}, \ldots, \mathrm{A}_{\mathrm{m}}$ whose probabilities of occurrence $p_{1}, \ldots, p_{m}$ are known. Entropy is defined as:

$$
H=-K \sum_{i=1}^{m} p_{i} \log \left(p_{i}\right)
$$

We can calculate the amount of information there is in an event using the probability of the event. This is called "Shannon information," "self-information," or simply the "information," and can be calculated for a discrete event $\mathrm{x}$ as follows:

$$
\operatorname{information}(x)=-\log (p(x))
$$

Where $\log ()$ is the base- 2 logarithm and $\mathrm{p}(\mathrm{x})$ is the probability of the event $\mathrm{x}$. The choice of the base- 2 logarithm means that the units of the information measure are in bits (binary digits). This can be directly interpreted in the information processing sense as the number of bits required to represent the event. We calculate the payload entropy value as defined above. The payload entropy value is calculated as the amount of information in the packet for it to be identified uniquely. For the remaining features, the parsing script examines individual packets and all the features belonging to the packet are extracted, processed and saved as a fingerprint file. All feature properties are initially assigned a value of 0 . These features are then queried in the packet. If the features have corresponding data, the data is processed in the variable, otherwise, the value of the variable remains as 0 .

\section{Selection of Optimal Feature Set and Machine Learning Algorithm}

The second stage of our proposed methodology (see Fig. 1) is the selection of the optimal feature set and the machine 
Proposed Methodological Framework

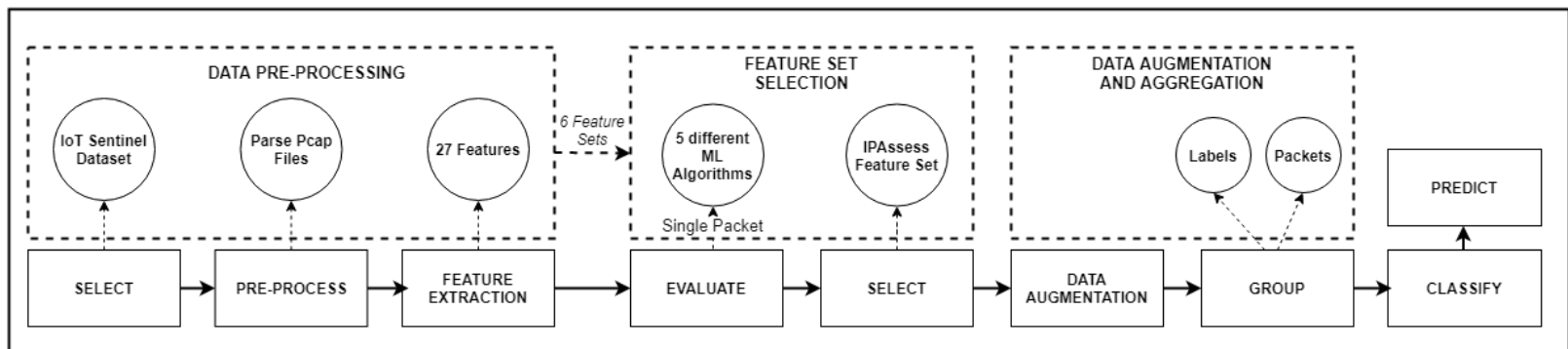

Fig. 1. Representation of the complete methodology proposed for IoT device classification

learning algorithm which provides the highest classification accuracy. We identified feature sets based on the implementation from three renowned studies [9] [13] [12] in the IoT device identification field (see Fig. 2). We proposed two novel feature sets based on the correlation matrix formed among the various protocols. To obtain an efficient feature set, we set the threshold value as 0.03 . Hence, if the correlation between two features is equal to or more than 0.03 , we decided to include it in the feature set. We perform an in-depth experimental analysis survey on five machine learning algorithms namely, Naive Bayes(NB), DT, RF, K-Nearest Neighbor (KNN) and Gradient Boost(GB), with accuracy and the time taken as the evaluation metric. For each feature set, the following metrics are displayed, accuracy, precision, recall, F1 score, kappa, training time, testing time and weighted average. We computed the results on the main dataset, with the original amount of features, without extracting any features. We then test the algorithms on the IoT Sentinel feature set, which comprises of the 23 features out of the original 26 features present in the dataset. This feature set consists of 16 protocol based features which are typically used during device association over WiFi, 2 binary features representing the use of IP header options, size of packet and presence of raw data, destination address mapped to a counter, IP padding and router alert, and two features representing the source and destination ports. None of the features rely on packet payload ensuring that fingerprints can be extracted from encrypted traffic. [9]

We decided to implement the feature set proposed in the IoTSense paper [13]. According to this study, they used many protocol based features, but restrained from using IP addresses and target and source port numbers. This was done to reduce the features which are too specific and therefore would be ineffective to distinguish between device behaviour. In addition to these features, this feature set also includes features related to payload, which are payload length, entropy of payload and TCP window size. They used payload length instead of packet size feature.

The final feature set was the implementation of the IoT DevID study [12]. The feature set was formulated without retaining the IP addresses and port number related features, as it was discovered on closer inspection that IoT Sentinel feature set did not use IP addresses and port numbers directly but rather used a counting process to summarise this information. This meant that the data did not capture unnecessary and overly-specific information while being able to obtain diversity. Also, they did not use payload length as there was a high correlation between packet size and payload length.

In addition to the aforementioned feature sets we also formulated our own feature sets on the basis of correlation with respect to two protocols namely, ARP (Address Resolution Protocol) and IP (Internet Protocol). We selected ARP as it is one of the main protocols used for mapping IP addresses to MAC addresses and IP is widely used as a principal communication protocol.

The ARP feature set discarded the following features: LLC, EAPOL, ICMP, ICMPv6, UDP, TCP_w_size, NTP, IP_padding, IP_ralert and Payload_l as they did not show much correlation with the threshold value being set as 0.03 . Similarly for the IP feature set the following features were discarded: ICMP, DHCP, BOOTP, SSDP, DNS, MDNS, NTP and Packet_raw_data. For these six feature sets, we observed the performance of each algorithm and hence chose the best algorithm and the feature set. Our experimental analysis was to survey the highest accuracy achieved along with the time taken into account with the least amount of features present.

\section{Data Augmentation}

The main concern which we discovered during the course of our research was the limited amount of data present, therefore we implemented the technique of data augmentation which expanded the size of the data present in the selected feature set. This increased the diversity of data significantly, which helps in efficiently training the model. It also took care of the problem of underfitting where in the model does not have to skip learning the features which are scarcely present.

Resampling is an important part of augmenting the data. This was done by using a Python library named scikit-learn, consisting of a utility named resample. Using this, the dataset is divided into training and testing data. We set the ratio of division as $0.75: 0.25$, which means $75 \%$ training data and $25 \%$ testing data. Due to the presence of imbalanced classes, we chose the Up-sample Minority Class method, to address the issue. In this process, the observations present in the minority class, are randomly duplicated. Therefore, all the classes have an equal opportunity to be represented and hence to be predicted by the model. 


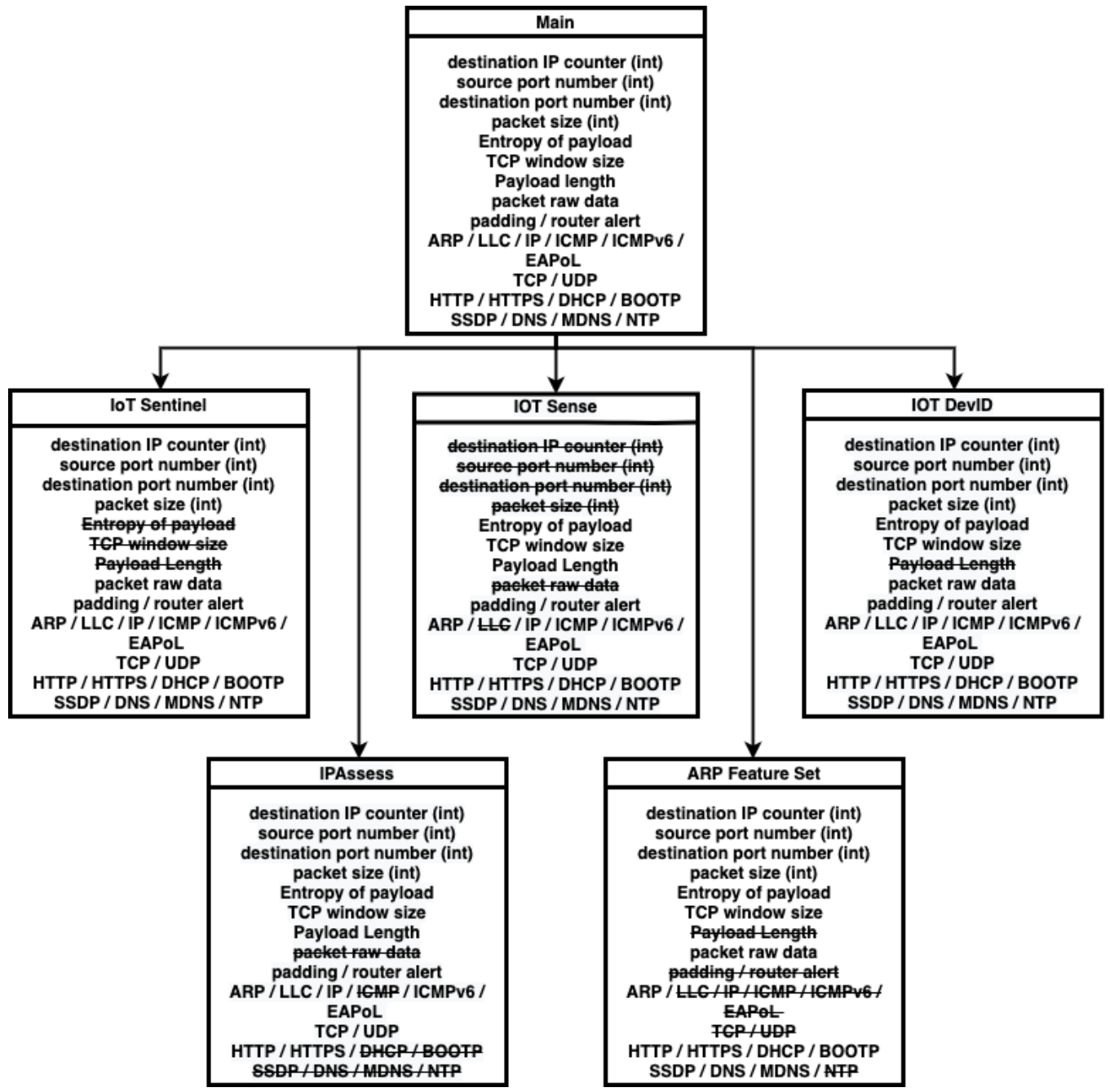

Fig. 2. Representing the relation of the feature sets formed

\section{E. Data Aggregation}

For the classification of different IoT devices, we propose the method of classification based on the grouping of packets. We have different group sizes of 1, 3, 6, 9, 12 and 100 packets, which means that the algorithm receives a group of packets from a particular device then the classification of the particular device is performed. This process continues for a total of 100 iterations for each of the devices with varying group sizes of packets in order to determine the efficient group size of packets that should be used for the classification. Through this process, we find out the optimal number of packets that should be received from a device in order to classify it with a higher accuracy. After observing the results of the above proposed method, we decided to combine devices into grouped labels along with the grouping of packets. In this, we aggregated and formed common labels for similar types of devices. This technique resulted in saving computation power as the number of labels in which a particular device needs to be classified is further reduced. As shown in the algorithm (see Algorithm 1) we take the MAC addresses of the devices in the dataset $\mathrm{M}$ $=m_{1}, m_{2}, \ldots, m_{n}$ and the result of the machine learning algorithm $\mathrm{Y}=y^{1}, y^{2}, \ldots, y^{n}$ as input. It creates groups of size $g$ from packets grouped according to MAC addresses. We first create a two-dimensional array to store the different devices present in the dataset.A variable seen is initialized and the group size is mentioned for the particular aggregation step. For each of the MAC addresses belonging to the devices is found and added to the seen list. It is then assigned a position in the two dimensional array and packets are then allotted to the machine learning algorithms as mentioned in the paper. We then divide the packets into the chunks of data given by the value of $g$ and the particular machine learning algorithms are provided with their chunks of data for experimental analysis.

\section{F. Prediction Model}

We tested out a prediction-based classification model formulated by combining the optimal machine learning algorithms and the feature set from the aforementioned experiments on the IoT Sentinel Dataset. The prediction-based classification system works by initially generating a list of all the pcap files using a generator function. It then, uses another generator function to filter out the packets based on the MAC addresses of the particular devices. Based on the optimal feature set found, we use the retained features and extract said fea- 


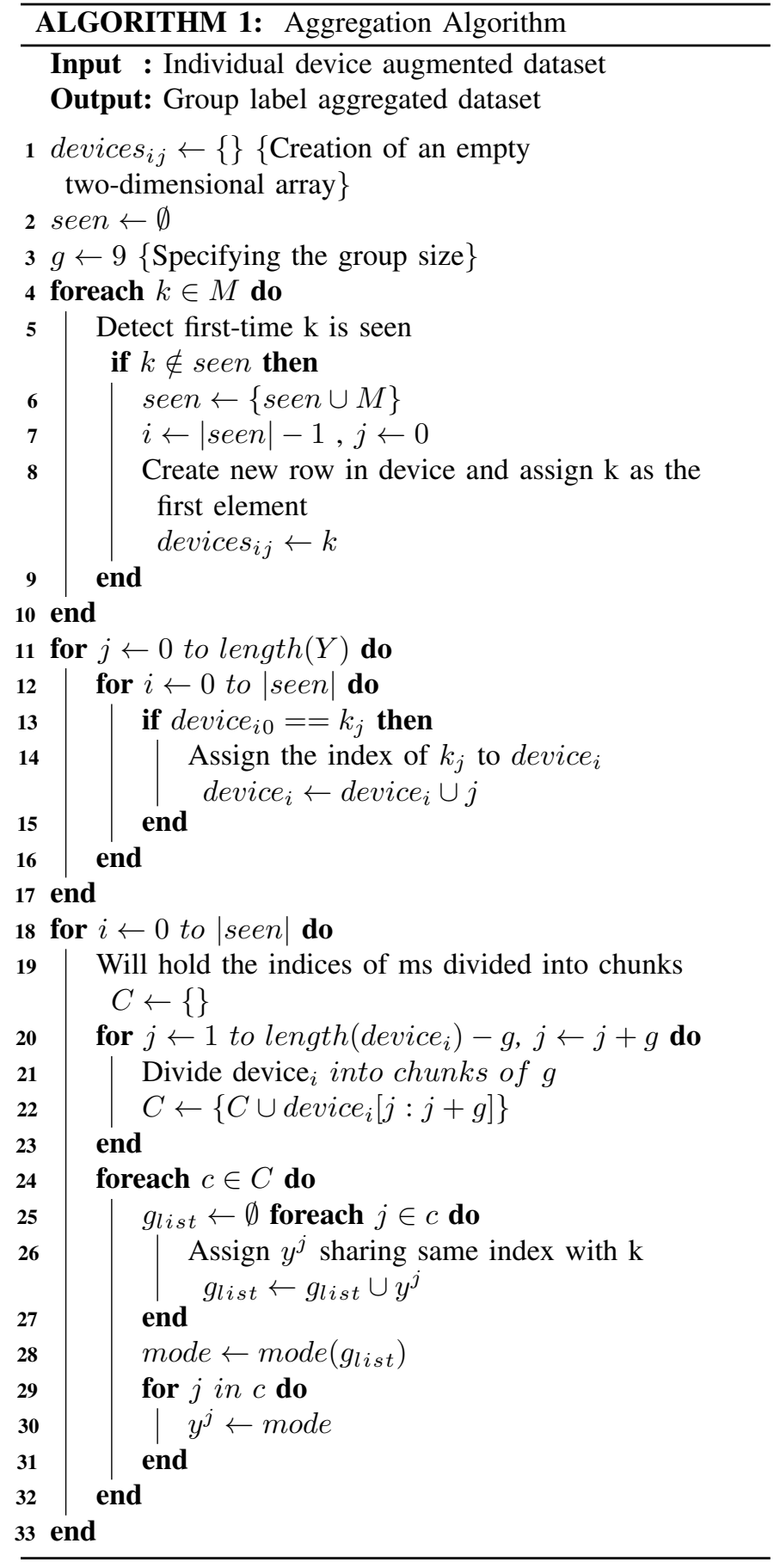

tures from the network packet captures. The model generates the complete dataset with 276 dimensional feature vectors. Thus, we extract 16 unique features for each device type concatenated into a 276 dimensional vector. We calculate the Damerau-Levenshtein Distance [15], which is a string metric for measuring the edit distance between two given sequences of data.

The classification model then calculates the feature importance of all devices using sklearn classifiers. Using the Damerau-Levenshtein edit distance and the classification prediction given by model, we are able to plot a graph on how the model performed with respect to the data provided in the dataset.

\section{RESULTS AND DISCUSSION}

With the implementation of the above algorithmic study, we start with the results obtained by the experimental analysis of the above discussed machine learning algorithms on the six different features.

The accuracy and $F_{1}$ score of each ML algorithm, when applied on a particular feature set, is shown in Table II. For the Main feature set which has all the features of the IoT devices, we find RF having the highest classification accuracy with $78.4 \%$ whereas DT has a higher F-1 score. KNN and DT have an accuracy of $76.8 \%$ and $77.9 \%$ respectively which is closer to RF as opposed to Extra Gradient Boost and Naive Bayes which perform poorly with $45.5 \%$ and $19.5 \%$ accuracy respectively. On the basis of $F_{1}$ score, DT and RF are the best performing algorithms.

For the IoT Sentinel feature set which has the features set up according to the IoT Sentinel method [9], we see that RF and DT have high accuracies of $72.4 \%$ and $72.1 \%$ as well as high $F_{1}$ scores of $62.7 \%$ and $62.6 \%$ respectively. KNN has an accuracy of $70.2 \%$ which is closer to RF and DT as opposed to Extra Gradient Boost and Naive Bayes which perform poorly with $42.5 \%$ and $36.6 \%$ accuracy respectively. This feature set did not perform as well as the Main feature set which is why we sought to discard this in the next part of our implementation.

For the IoTSense feature set which has the features of the IoT devices according to the IoTSense method [13], we see that all the algorithms perform poorly in comparison, with RF having the highest accuracy of $68.9 \%$ and highest $F_{1}$ score of $55.2 \%$. KNN and DT have an accuracy of $67.4 \%$ and $68.6 \%$ respectively which were closer to RF as opposed to Extra Gradient Boost and Naive Bayes which perform extremely poorly with $36.9 \%$ and $5.6 \%$ accuracy respectively. This feature set did not perform as well as the Main feature set which is why we sought to discard this in the next part of our implementation.

For the IoTDevID feature set which was formulated with the features according to the IoTDevID method [12], we study that RF and DT has the same accuracy of $77.5 \%$ but DT has a higher $F_{1}$ score of $67.6 \%$, hence making DT a better algorithm in this case. While KNN has an accuracy of $76.1 \%$ which was closer to RF as opposed to Extra Gradient Boost and Naive Bayes which perform poorly with $44.8 \%$ and $22.4 \%$ accuracy respectively. This feature set did not perform as well as the Main feature set which is why we sought to discard this in the next part of our implementation.

For the proposed ARP feature set having the features of the IoT devices which has a high correlation with the ARP protocol layer, we see that RF has the highest accuracy with $72.8 \%$ while RF and DT have an equal and highest $F_{1}$ score of $62.9 \%$. KNN and DT have an accuracy of $70.6 \%$ and $72.5 \%$ respectively which were closer to RF as opposed to Extra Gradient Boost and Naive Bayes which under perform with $44.5 \%$ and $36.1 \%$ accuracy respectively. This feature set did not perform as well as the Main feature set, however 
TABLE II

Performance of Ml Algorithms on each Feature Set formed

\begin{tabular}{c|cccccccccc}
\hline & \multicolumn{5}{|c|}{ Accuracy } & \multicolumn{4}{c}{ F1 Score } \\
\hline Feature Sets & RF & DT & KNN & NB & XGB & RF & DT & KNN & NB & XGB \\
\hline \hline Main Feature Set & $78.4 \%$ & $77.9 \%$ & $76.8 \%$ & $19.5 \%$ & $45.5 \%$ & $67.5 \%$ & $67.8 \%$ & $66.0 \%$ & $45.6 \%$ & $7.5 \%$ \\
\hline IoT Sentinel Feature Set & $72.4 \%$ & $72.1 \%$ & $70.2 \%$ & $36.6 \%$ & $42.5 \%$ & $62.7 \%$ & $62.6 \%$ & $60.1 \%$ & $22.5 \%$ & $9.8 \%$ \\
\hline IoT Sense Feature Set & $68.9 \%$ & $68.6 \%$ & $67.4 \%$ & $5.6 \%$ & $36.9 \%$ & $55.2 \%$ & $54.9 \%$ & $54.2 \%$ & $3.06 \%$ & $6.8 \%$ \\
\hline IoT DevID Feature Set & $77.5 \%$ & $77.5 \%$ & $76.1 \%$ & $22.4 \%$ & $44.8 \%$ & $67.1 \%$ & $67.6 \%$ & $65.5 \%$ & $8.8 \%$ & $11.3 \%$ \\
\hline ARP Feature Set & $72.8 \%$ & $72.5 \%$ & $70.6 \%$ & $36.1 \%$ & $44.5 \%$ & $62.9 \%$ & $62.9 \%$ & $61.1 \%$ & $21.8 \%$ & $11.6 \%$ \\
\hline IPAssess Feature Set & $78.3 \%$ & $78.0 \%$ & $76.9 \%$ & $19.1 \%$ & $47.9 \%$ & $67.9 \%$ & $66.3 \%$ & $66.9 \%$ & $7.8 \%$ & $14.8 \%$ \\
\hline
\end{tabular}

there is an exponential increase in the Naive Bayes accuracy. Even though the basic assumption in Naive Bayes is that the features are considered to be independent, which is practically rarely possible. The performance of this algorithm depends on whether the correlated feature sets are good predictors or not. Hence, the model benefits if the correlated feature sets happen to be good predictors, as seen in this case. However, the overall accuracies and $F_{1}$ scores are lower which is why we sought to discard this in the next part of our implementation.

For the proposed IPAssess feature set which has the features of the IoT devices according to the correlation method with IP protocol, we see that RF and DT have high accuracies of $78.3 \%$ and $78.0 \%$ where RF has the highest $F_{1}$ score of $67.9 \%$, amongst all the feature sets and algorithms used. KNN has an accuracy of $76.9 \%$ which was closer to RF as opposed to Extra Gradient Boost and Naive Bayes which perform poorly with $47.9 \%$ and $19.1 \%$ accuracy respectively. We selected this feature set as it has nine less features than the main feature set, giving an accuracy similar to it which proves that it was the optimal feature set to test our algorithm on. This helps in discarding insignificant features, thus making it less complex for the algorithm used on that particular feature set.

In all of the above feature sets we can observe that $\mathrm{KNN}$ is performing better than Naive Bayes and Extra Gradient Boost in terms of accuracy, but the only backdrop is the classification time. KNN takes more time than any other algorithm, which is mostly due to the fact that KNN is a lazy learner which means that there is no training required before making predictions, thus it does all the calculations while making predictions. We can even notice that RF Classifier and DT have a close competition to become the best classifier in terms of accuracy, $F_{1}$ score and time. The reason for RF classifier performing better than DT is that the former chooses the features randomly instead of relying on a specific set of features unlike the latter which is during the training time. RF is comparatively more robust and thus also prevents the problem of over-fitting while giving the result in a lesser amount of time. In some cases, DT outperforms RF in terms of $F_{1}$ score due to the basic explanation that DT focuses more on specific features rather than taking an average of the features selected by different decision trees when RF algorithm is applied on the same feature set.

The output also displays the precision, recall, $F_{1}$ score and support of each of the devices when classified using a particular ML algorithm on the selected feature set. In Table III, the above mentioned evaluation metrics are calculated for each of the device classified using Random Forest classifier, which was applied on IPAssess Feature Set.

After going through the results, we are confident to choose IPAssess as our feature set. The next stage of our proposed methodology was to augment the dataset to provide more robust data, expanding the size along with balancing the classes. With our selected IPAssess feature set, we do an experimental analysis with accuracy and time taken as the evaluation criteria. For a grouping of 3 packets (see Table IV), the accuracy for both the algorithms increase exponentially with $85 \%$ and $84.6 \%$ for RF and DT respectively. The accuracies increase with the group of 6 packets being taken with the accuracy recorded as $91.7 \%$ for RF Classifier. It further increased to $93.3 \%$ for a group of 9 packets. We see RF performing extraordinarily giving an accuracy of $95.7 \%$ for a group of 12 packets and DT having the highest baseline accuracy of $99.6 \%$ when a group of 100 packets are taken, trained and tested. Along with the accuracy we can also find the classification time taken by each particular algorithm (see Table VI). We see that DT takes the least amount of time while maintaining the classification accuracy.

Along with this augmentation and aggregation method we also combined labels, grouping common devices into a single label to test out the classification accuracy. The accuracy and the time taken for both non-combined labels and combined labels are presented in the following tables (see Table $\mathrm{V}$ and Table VII). With this grouping in place we see that both RF and DT's accuracy significantly increase for individual packets to being $88.9 \%$ and $88.5 \%$ respectively while DT's classification time decreases to 0.15 seconds. For a group of 3 packets, along with this methodology, RF and DT have almost a similar accuracy: $94.7 \%$ and $94.5 \%$ respectively. They further increase when a group of 6 packets are taken into consideration with both algorithms achieving a classification accuracy of $97.8 \%$. Both the algorithms gave optimal results with 98\% accuracy for both groups of 9 and 12 packets. In addition to this, we see KNN gives better accuracy or in-fact the highest accuracy amongst all the other ML algorithms when aggregated with combined labels in a group of 6,9 and 12 packets with $97.9 \%, 98.2 \%$ and $98.9 \%$ respectively. However, with KNN's time being almost 4 times RF's and 240 times DT's classification time, it is not reliable in a realworld application prediction model. As we can see, DT gives us a close enough accuracy to RF and takes as little as 0.3 seconds to finish an iteration. When we combine labels, DT 
TABLE III

Performance of RF Classifier on Different Devices using the IPAssess Feature Set

\begin{tabular}{ccccc}
\hline Devices & Precision & Recall & F1 Score & Support \\
\hline \hline Aria & 0.8484 & 0.7627 & 0.7983 & 52.0 \\
\hline D-LinkCam & 0.8688 & 0.8935 & 0.8809 & 635.8 \\
\hline D-LinkDayCam & 0.9047 & 0.7916 & 0.8436 & 123.5 \\
\hline D-LinkDevice & 0.7857 & 0.8183 & 0.8016 & 1067.8 \\
\hline D-LinkSensor & 0.4983 & 0.4787 & 0.4880 & 663.3 \\
\hline D-LinkSiren & 0.4264 & 0.3470 & 0.3823 & 628.9 \\
\hline D-LinkSwitch & 0.6676 & 0.6083 & 0.6363 & 661.4 \\
\hline D-LinkWaterSensor & 0.4730 & 0.5066 & 0.4889 & 653.8 \\
\hline EdimaxCam & 0.9091 & 0.7986 & 0.8492 & 89.6 \\
\hline EdimaxPlug1101W & 0.54022 & 0.5175 & 0.5268 & 124.7 \\
\hline EdimaxPlug2101W & 0.4608 & 0.4262 & 0.4404 & 113.1 \\
\hline EdnetCam & 0.8187 & 0.6093 & 0.6941 & 39.0 \\
\hline EdnetGateway & 0.8243 & 0.6708 & 0.7378 & 85.0 \\
\hline HomeMaticePlug & 1.0 & 0.9562 & 0.9775 & 63.9 \\
\hline Hue-Device & 0.9817 & 0.9891 & 0.9854 & 3258.1 \\
\hline Lightify & 0.6260 & 0.9753 & 0.7624 & 438.4 \\
\hline MaxGateway & 0.9490 & 0.8745 & 0.9097 & 63.4 \\
\hline SmarterCoffee & 0.2503 & 0.1402 & 0.1724 & 19.0 \\
\hline TP-LinkPlugHS100 & 0.6157 & 0.5146 & 0.5584 & 72.9 \\
\hline TP-LinkPlugHS110 & 0.5802 & 0.5555 & 0.5644 & 69.8 \\
\hline WeMoInsightSwitch & 0.7972 & 0.8111 & 0.8038 & 607.7 \\
\hline WeMoLink & 0.9223 & 0.8389 & 0.8785 & 676.9 \\
\hline WeMoSwitch & 0.7774 & 0.7801 & 0.7785 & 460.7 \\
\hline Withings & 0.9035 & 0.7460 & 0.8158 & 77.7 \\
\hline iKettle2 & 0.1810 & 0.0904 & 0.1141 & 18.8 \\
\hline & & & &
\end{tabular}

TABLE IV

Accuracy Recorded When Not Aggregated With Combined Labels

\begin{tabular}{cccccc}
\hline \multicolumn{7}{c}{ IPAssess Feature Set } \\
\hline Group & NB & DT & RF & XGB & KNN \\
\hline \hline 1 & 19.8 & 77.6 & 78.1 & 48.3 & 76.5 \\
\hline 3 & 18.7 & 84.6 & 85 & 52.1 & 83.2 \\
\hline 6 & 23.9 & 91.4 & 91.7 & 54.7 & 90.9 \\
\hline 9 & 26.3 & 93.1 & 93.3 & 55.6 & 92.1 \\
\hline 12 & 29.1 & 95.1 & 95.7 & 56.1 & 88.9 \\
\hline 100 & 35.4 & 99.6 & 99.5 & 57.8 & 98.9 \\
\hline
\end{tabular}

TABLE V

Accuracy Recorded When Aggregated With Combined Labels

\begin{tabular}{cccccc}
\hline \multicolumn{7}{c}{ Combined Labels } \\
\hline Group & NB & DT & RF & XGB & KNN \\
\hline \hline 1 & 20.2 & 88.5 & 88.9 & 57.9 & 87.3 \\
\hline 3 & 20.1 & 94.5 & 94.7 & 62.1 & 94.3 \\
\hline 6 & 25.5 & 97.8 & 97.8 & 63.3 & 97.9 \\
\hline 9 & 27 & 98.2 & 98.1 & 63.2 & 98.2 \\
\hline 12 & 29.9 & 98.8 & 98.8 & 63.8 & 98.9 \\
\hline
\end{tabular}

takes a constant time of 0.15 seconds, making it the most optimal algorithm to make a model out of. Since RF has to create many decision trees on the basis of choosing features randomly, the DTs take much less time as it has already been trained on the IPAssess feature set and it makes the decision on the basis of a specific set of features.

Even though, KNN has got the highest accuracy in the proposed technique of device classification, the time consumed
TABLE VI

Time Taken (in sec) When Not Aggregated With Combined Labels

\begin{tabular}{cccccc}
\hline \multicolumn{7}{c}{ IPAssess Feature Set } \\
\hline Group & NB & DT & RF & XGB & KNN \\
\hline \hline 1 & 0.2 & 0.45 & 7.5 & 27 & 69 \\
\hline 3 & 0.25 & 0.25 & 4 & 14 & 33 \\
\hline 6 & 0.3 & 0.3 & 4 & 14 & 33 \\
\hline 9 & 0.25 & 0.25 & 4 & 15 & 33 \\
\hline 12 & 0.3 & 0.3 & 6.5 & 17 & 45 \\
\hline 100 & 0.3 & 0.3 & 3.5 & 15 & 35 \\
\hline
\end{tabular}

TABLE VII

Time Taken (in sec) When Aggregated With Combined Labels

\begin{tabular}{cccccc}
\hline \multicolumn{7}{c}{ Combined Labels } \\
\hline Group & NB & DT & RF & XGB & KNN \\
\hline \hline 1 & 0.15 & 0.15 & 4 & 10 & 36 \\
\hline 3 & 0.05 & 0.15 & 3 & 10 & 39 \\
\hline 6 & 0.15 & 0.15 & 3 & 10 & 38.5 \\
\hline 9 & 0.2 & 0.15 & 3.5 & 10 & 38 \\
\hline 12 & 0.2 & 0.15 & 3.5 & 10 & 38 \\
\hline
\end{tabular}

by this algorithm is around 38 seconds which is way more than any other algorithm. This is due to the fact that KNN does not require any training, hence it does all the computation during the testing period.

The supervised learning algorithms work best on linearly separable classes, hence we merged the similar kind of devices into one label, naming it combined labels. This helps to increase the performance of the algorithm due to the reduced 
number of overlapping classes.

The results show that these algorithms perform better when aggregated with combined labels. The reason is that all these algorithms are supervised algorithms, hence it shows better accuracy and consumes lesser time than the other.

We observe from the results that KNN algorithm performs better than Random Forest and Decision Tree in a group of 6 to 12 packets when we aggregate combined labels. This can be explained on the basis of the principle a particular algorithm works. KNN works on the principle of similarity measure. Hence, we can see that the accuracy increases when the algorithm receives a group of 6 packets and not when its classifying devices as soon as it receives 1 or 3 packets from the same device.

Random Forest classifier works well in most of the cases. This stems from the fact that the model predicts the class of the device on the basis of the majority of the class predicted by each of the individual trees in random forest. It usually works better than Decision Tree as the latter considers each and every possible feature to split the node in order to produce the most separation. On the other hand, a random subset of features is picked by an individual tree in Random Forest to split the node. This helps to save the individual trees from their possible errors as its a collective prediction.

\section{CONCLUSION}

In this research, we investigated device recognition and classification based on network traffic analysis from the packets captured and dataset generated by the IoT Sentinel dataset. We successfully developed and implemented our approach that involved data pre-processing, followed by selection of the optimal feature set and then testing the dataset on five machine learning algorithms.

We see improved and comparable performance in our results based on measurement of accuracy and the time taken by each of the algorithms, achieved by the process of reducing the number of features set. Additionally, efficiency was achieved by normal grouping and then combined label grouping.

The integration of various machine learning algorithms seems to be the next step in the classification field. We plan to explore merging machine learning algorithms after testing them out individually to enhance the efficiency and accuracy in classification. One of the other future work would involve investigating the possibility of classifying the IoT devices in real time, which means if a device is not present in the training data, the model will simultaneously learn about the new device and classify it accordingly.

\section{REFERENCES}

[1] Q. Jing, A. Vasilakos, J. Wan, J. Lu, and D. Qiu, "Security of the internet of things: Perspectives and challenges," Wireless Networks, vol. 20, pp. 2481-2501, 112014.

[2] M. R. Shahid, G. Blanc, Z. Zhang, and H. Debar, "Iot devices recognition through network traffic analysis," in 2018 IEEE international conference on big data (big data). IEEE, 2018, pp. 5187-5192.

[3] S. Agarwal, P. Oser, and S. Lueders, "Detecting iot devices and how they put large heterogeneous networks at security risk," Sensors, vol. 19, no. 19 , p. $4107,2019$.
[4] A. Mavrogiorgou, A. Kiourtis, and D. Kyriazis, "Iot devices recognition through object detection and classification techniques," in 2019 Third World Conference on Smart Trends in Systems Security and Sustainablity (WorldS4). IEEE, 2019, pp. 12-20.

[5] A. Sivanathan, H. H. Gharakheili, and V. Sivaraman, "Managing iot cyber-security using programmable telemetry and machine learning," IEEE Transactions on Network and Service Management, vol. 17, no. 1, pp. $60-74,2020$.

[6] Y. Meidan, M. Bohadana, A. Shabtai, J. D. Guarnizo, M. Ochoa, N. O. Tippenhauer, and Y. Elovici, "Profiliot: A machine learning approach for iot device identification based on network traffic analysis," in Proceedings of the symposium on applied computing, 2017, pp. 506509.

[7] I. Alrashdi, A. Alqazzaz, E. Aloufi, R. Alharthi, M. Zohdy, and H. Ming, "Ad-iot: Anomaly detection of iot cyberattacks in smart city using machine learning," in 2019 IEEE 9th Annual Computing and Communication Workshop and Conference (CCWC). IEEE, 2019, pp. 0305-0310.

[8] A. Aksoy and M. H. Gunes, "Automated iot device identification using network traffic," in ICC 2019-2019 IEEE International Conference on Communications (ICC). IEEE, 2019, pp. 1-7.

[9] M. Miettinen, S. Marchal, I. Hafeez, N. Asokan, A.-R. Sadeghi, and S. Tarkoma, "Iot sentinel: Automated device-type identification for security enforcement in iot," in 2017 IEEE 37th International Conference on Distributed Computing Systems (ICDCS). IEEE, 2017, pp. 21772184.

[10] R. R. Chowdhury, S. Aneja, N. Aneja, and E. Abas, "Network traffic analysis based iot device identification," in Proceedings of the 2020 the 4th International Conference on Big Data and Internet of Things, 2020, pp. 79-89.

[11] L. Bai, L. Yao, S. S. Kanhere, X. Wang, and Z. Yang, "Automatic device classification from network traffic streams of internet of things," in 2018 IEEE 43rd conference on local computer networks (LCN). IEEE, 2018, pp. $1-9$.

[12] K. Kostas, M. Just, and M. A. Lones, "Iotdevid: A behaviour-based fingerprinting method for device identification in the iot," arXiv preprint arXiv:2102.08866, 2021

[13] B. Bezawada, M. Bachani, J. Peterson, H. Shirazi, I. Ray, and I. Ray, "Iotsense: Behavioral fingerprinting of iot devices," arXiv preprint arXiv:1804.03852, 2018.

[14] C. E. Shannon, "A mathematical theory of communication," The Bell system technical journal, vol. 27, no. 3, pp. 379-423, 1948.

[15] F. P. Miller, A. F. Vandome, and J. McBrewster, "Levenshtein distance: Information theory, computer science, string (computer science), string metric, damerau? levenshtein distance, spell checker, hamming distance," 2009.

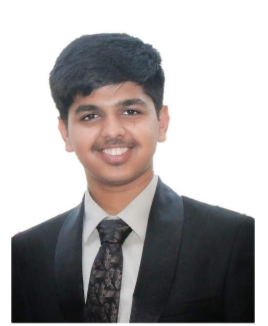

Parth Ganeriwala is a Research Professional at the Computer Engineering and Sciences Department, Florida Institute of Technology. He is also a senior student pursing his undergraduate in Computer Science at BITS Pilani, Dubai Campus. His research interests are in the area of Deep Learning, Cyber Security and Robotics.

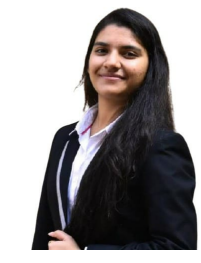

Shreya Nandanwar is in her senior year of the undergraduate degree in Computer Science department at BITS Pilani Dubai Campus. She is currently carrying out research in the area of Explainable AI under the guidance of Prof. Siddhartha Bhattacharya and Dr. Raja Muthalagu. She has earlier researched in the area of Neuromorphic Computing. Her interest lies in the field of Human - Computer interaction. 


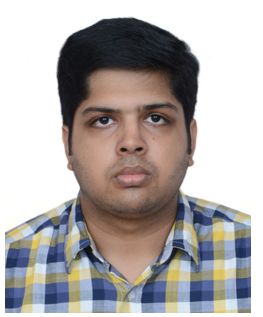

Anubhav Gupta is a Master's student at the CMPS Department, University of British Columbia. He received his B.E in Computer Science from BITS Pilani, Dubai Campus. His research interests are in the area of Intelligent Autonomous Systems, Deep Learning, and Formal Methods. In his free time, he loves to play chess.

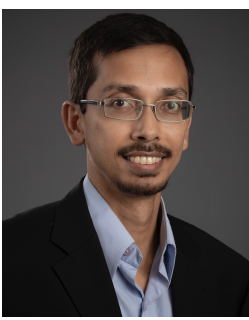

Dr. S Bhattacharyya is a faculty at Florida Institute of Technology. His research lab ASSIST (Assured Safety Security and Intent with Systematic Tactics) focuses on research in the area of formal methods for the design, verification and validation of intelligent autonomous systems, avionics, cyber security, IOT and systems biology. Previously, he had been a Sr. Research Engineer at Rockwell Collins' Advanced Technology Center where he worked on research programs for assurance of safety critical systems.

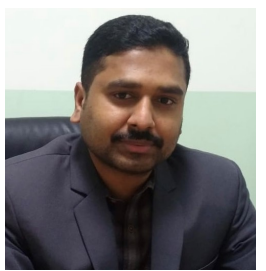

Dr. Raja Muthalagu is a faculty at BITS Pilani, Dubai Campus. He primarily conducts research in the area of Wireless Communications, Signal Processing, IoT and cyber security. He was a postdoctoral research fellow at ATMRI, Nanyang Technological University (NTU), Singapore during 2014-2015. He was a recipient of Canadian Commonwealth Scholarship Award-2010 for Graduate Student Exchange Program in the Department of Electrical and Computer Engineering, University of Saskatchewan, Saskatoon, Canada. 